\title{
Free Fatty Acids Quantification in Dairy Products
}

\author{
Kieran N. Kilcawley and David T. Mannion \\ Additional information is available at the end of the chapter
}

http://dx.doi.org/10.5772/intechopen.69596

\begin{abstract}
Quantification of free fatty acids in dairy products is not only important due to their (fatty acids) impact on the flavour and texture of dairy products but also because of their potential impact on nutrition and health, and as anti-microbial agents. This chapter provides an overview of the practical issues associated with existing lipid extraction techniques and quantification procedures using gas chromatography flame-ionization detection. The most widely used methods are compared and recent advancements in the quantification of free fatty acids in dairy products are discussed.
\end{abstract}

Keywords: free fatty acids, dairy, gas chromatography

\section{Introduction}

Bovine milk fat has a complex fatty acid composition with over 400 individual fatty acids $[1,2]$. However, only 15 or 16 fatty acids are present in milk at concentrations above $1 \%$ [3, 4]. The predominant fatty acids have a straight carbon atom chain with an even number of carbons and may be either saturated or unsaturated [5]. The approximate composition of the fatty acids of bovine, ovine and caprine milk fat is given in Table 1 . The proportion of fat in bovine, ovine and caprine milk is $\sim 3.8,7.1$ and $3.7 \%$, respectively, and the fatty acid composition of milk within cows, goats and sheep milk is influenced by diet, stage of lactation and breed among others [6]. Consequently, the proportions of some fatty acids can show marked variations.

Animal fats are complex and contain short-chain fatty acids (SCFFA) that are both water soluble and highly volatile and are not present in vegetable fats [3]. Vegetable fat consists of primarily non-volatile fat-soluble fatty acids. The main fatty acids in milk are $\mathrm{C}_{16: 0}$ and $\mathrm{C}_{18: 1}$ 


\begin{tabular}{|c|c|c|c|c|}
\hline Fatty acids (g $\left.1100 \mathrm{~g}^{-1}\right)$ & Common name & Bovine & Ovine & Caprine \\
\hline $\mathrm{C}_{4: 0}$ & Butyric acid & 2.87 & 2.57 & 2.03 \\
\hline $\mathrm{C}_{6: 0}$ & Caproic acid & 2.01 & 1.87 & 2.78 \\
\hline $\mathrm{C}_{8: 0}$ & Caprylic acid & 1.39 & 1.87 & 2.92 \\
\hline $\mathrm{C}_{10: 0}$ & Capric acid & 3.03 & 6.63 & 9.59 \\
\hline $\mathrm{C}_{12: 0}$ & Lauric acid & 3.64 & 3.99 & 4.52 \\
\hline $\mathrm{C}_{14: 0}$ & Myristic acid & 10.92 & 10.17 & 9.83 \\
\hline $\mathrm{C}_{16: 0}$ & Palmitic acid & 28.7 & 25.1 & 24.64 \\
\hline $\mathrm{C}_{18: 0}$ & Stearic acid & 11.23 & 8.85 & 8.87 \\
\hline $\mathrm{C}_{18: 1}$ cis-9 & Oleic acid & 22.36 & 20.18 & 18.65 \\
\hline $\mathrm{C}_{18: 2}$ cis-9, cis-12 & Linoleic acid & 2.57 & 2.32 & 2.25 \\
\hline $\mathrm{C}_{18: 2}$ cis-9, trans -11 & Conjugated linoleic acid & 0.57 & 0.76 & 0.45 \\
\hline $\mathrm{C}_{18: 3}$ cis-9, cis-12, cis-15 & $\alpha$-Linolenic acid & 0.5 & 0.92 & 0.77 \\
\hline
\end{tabular}

Source: Adapted from Markiewick-Keszykca et al. [6].

Table 1. Distribution of the major fatty acids in bovine, ovine and caprine milk.

comprising between $\sim 22-35$ and $20-30 \%$ of total lipids, respectively [3]. Fatty acids are present either in their free state, as free fatty acids (FFA) or esterified as bound fatty acids (FA) on the glycerides. Accurate determination of both FFA and FA can be indispensable for legislative and quality control purposes but also for research and development purposes. This chapter focuses on gas chromatographic detection of FFA(s). FFA(s) are important as they influence product quality, flavour, texture, nutrition, and health. The flavour of many dairy products is directly and indirectly influenced by the FFA profile of the product $[2,7]$. This is particularly the case for fermented dairy products, as FFA contributes directly as volatile aromatic components or indirectly via volatile products of metabolism, oxidation or heat treatments (e.g. aldehydes, ketones, alcohols, lactones and esters). FFAs can also contribute to texture and functionality, as they impact on surface tension and foaming capacity of milk $[8,9]$, but some FFAs such as conjugated linoleic acid have also been shown to have beneficial health and nutritional effects [6].

Fat extraction techniques need to be able to take into account differences in solubility and volatility of the different carbon chain lengths of FA(s) present in milk fat. Therefore, any method for the accurate quantification of FA(s) must be efficient in extracting both water soluble SCFFA and organic soluble FA, avoid the use of evaporation steps to prevent losses of volatile SCFFA and remove or negate any water that may be present in the sample. So far, the most common approach to quantify FFA in the dairy industry and in research is gas chromatography (GC) coupled to a flame ionization detector (FID). FID is used because it is relatively cheap, simple/robust, reproducible and widely available. This chapter discusses in detail the advantages and disadvantages of some of the methods used to extract and quantify FFA in dairy products by GC-FID. 


\section{Free fatty acid determination in dairy products}

\subsection{Lipid extraction}

Solvent extraction of the fat from the sample is widely used. However, as previously mentioned, evaporation steps should be avoided to prevent losses of volatile SCFFA. Solvents such as chloroform [10], acidified diethyl ether [11], hexane/diethyl ether [12], and diethyl ether/heptane [13] have been used. High recoveries ( $>92 \%$ ) of FFA have been achieved using these organic solvents for cheese but are much less reliable when applied to milk, due to the natural oil in water emulsion of milk and the nature of the milk fat globule membrane (MFGM) [14]. In addition, extraction procedures that employ high temperatures such as refluxing or distillation are also prone to increased risk of losing volatile SCFFA(s). In many procedures, anhydrous sodium sulphate is added to absorb moisture present in an attempt to prevent losses of water-soluble SCFFA in the subsequent solvent extraction process. When solvent mixtures are employed, recoveries of SCFFA can decrease when the non-polar component of the solvent is increased [11]. Solvents capable of extracting the complete range of FFA(s) will also extract the remaining lipid portion of the sample and depending upon the application, it may be necessary or prudent to isolate the FFA component prior to analysis. A range of different techniques have been employed such as silicic acid/potassium hydroxide $(\mathrm{KOH})$ columns [10, 15, 16], ion exchange resins [17-19], deactivated alumina columns [12, 13] and aminopropyl solid phase extraction columns [13, 20].

Due to the strongly alkaline nature of silicic acid columns/KOH or ion exchange resins, hydrolysis of glycerides can occur [21,22] resulting in an overestimation of FFA content. Woo and Lindsay [16] implemented the use of two different columns, a pre-column to remove lactic acid followed by a silicic acid- $\mathrm{KOH}$ arrestant column to isolate FFA(s) in Cheddar cheese to overcome this issue. The FFA(s) were eluted using $2 \%$ formic acid in ethyl ether. Needs et al. [17] described another method to isolate FFA in milk, using a pre-treated amberlite resin. The lipid extract was mixed with the resin followed by solvent removal and washing to isolate the FFA. Deeth et al. [12] utilised deactivated alumina columns to isolate FFA and reported high recoveries of $\mathrm{C}_{4: 0}-\mathrm{C}_{18: 1}$ from milk, cheese and butter. The acidic nature of the final extract $(6 \%$ formic acid in di-isopropyl ether) was reported to have a detrimental impact on column performance as the column phase deteriorated and this leads to a modification of the procedure using a lower concentration of formic acid (3\%) in diethyl ether [13]. De Jong and Badings [13] using a reference mix compared the performance of aminopropyl columns and deactivated alumina in isolating FFA(s) and reported a 96-101\% recovery with aminopropyl columns against an $82-89 \%$ recovery for the deactivated alumina. The procedure was a modification of that employed by Kaluzny et al. [20] who obtained 101.4\% recovery for FFA(s) isolated from lipid using solid phase extraction with aminopropyl columns.

\subsection{Derivitization}

In order for an analyte to be analysed by GC, it must be volatile. For most lipids, this is not the case, and techniques using chemical derivitization are widely employed to volatilise the 
fatty acids. The most established approach is to convert the FA(s) into a more volatile form, such as fatty acid methyl esters, commonly known as FAME(s). Thus the FAME(s) are injected onto a GC column as a liquid volatilised into a gaseous mixture at a specified flow or pressure. Separation occurs through differences in the interaction of the individual FAME with the GC column phase and the use of a temperature gradient in the GC oven. The separated individual FAME passes from the column into the FID and is then quantified. A FAME approach is used in the current international standard for the analysis of FA(s) in milk fat [23]. FFA(s) can be converted into FAME(s) using methanol in the presence of a suitable acid catalyst. The first step is the protonation of the acid with methanol forming an intermediate, which loses a proton to yield the FAME. An excess of methanol is required to drive the reaction to completion as it is a reversible reaction. It is also necessary to exclude water from the reaction as it is a stronger electron donor than aliphatic alcohols and will inhibit the formation of the intermediate [24].

Several methods have been developed for FFA analysis of dairy products that derivitized at room temperature and do not employ aqueous solvents or evaporation steps. Christopherson and Glass [25] outlined the use of $10 \%$ hydrochloric acid $(\mathrm{HCl})$ in methanol to a solution of milk fat prior to GC analysis. Luddy et al. [26] used boron trifluoride $\left(\mathrm{BF}_{3}\right)$ in methanol to esterify FFA in butter oil. While these methods are useful, determination of FFAs is achieved by derivitizing FFA(s) with glycerides which involves two different derivitizing steps. Tetramethylammonium hydroxide (TMAH) has long been used as an esterification reagent for FA(s) [27]. Robb and Westbrook [28] identified that the TMAH reaction proceeds rapidly at room temperature, and that the salts readily decompose into the heated injection port of a GC to yield methyl esters and trimethylamine (TMA). The yields obtained ranged from 85 to $95 \%$, and it was concluded that these variable yields made the method suitable for qualitative purposes only. A limitation of many methylation procedures is the necessity to extract the acids from an aqueous solution prior to esterification. Downing [29] investigated the preparation of tetramethylammonium salts in an aqueous solution followed by GC analysis and found the method to be quantitative and reproducible. Downing and Greene [30] used TMAH to esterify polyunsaturated fatty acids (PUFAs) and found that the strong alkaline nature of the TMAH solution interfered with the esterification process. The authors overcome this by reducing the $\mathrm{pH}(7.5-8.0)$ using a 5\% acetic acid solution. An advantage of this approach was that the ammonium salts of FA(s) could be pyrolysed to form pure esters within the GC injection port as existing methods at the time required the saponification of the acids before the addition of esterifying agents. These methods described the saponification of FA mixtures with a strong base such as sodium hydroxide $(\mathrm{NaOH})$ or $\mathrm{KOH}$ followed by esterification with a methanolic acid such as $\mathrm{HCl}$, sulphuric acid $\left(\mathrm{H}_{2} \mathrm{SO}_{4}\right)$ or $\mathrm{BF}_{3}$ to determine FFA content. If the glyceride bound FA also required determination, a methanolic base such as sodium methoxide $\left(\mathrm{CH}_{3} \mathrm{NaO}\right)$ could be used to form the methyl esters. Since quaternary ammonium hydroxides are inherently strong bases, a methanolic solution can form methyl esters of the glyceride bound FA(s), and as the ammonium salts of FFA(s) degrade to form methyl esters, TMAH was considered to be a more suitable reagent in FAME analysis. Other quaternary ammonium hydroxides were also used in FAME analysis. McCreary et al. [31] used trimethyl (a,a,a-trifluoro-m-tolyl) ammonium hydroxide in methanol to determine 
the FA content of vegetable oils prepared in benzene. They compared this approach to the more common $\mathrm{CH}_{3} \mathrm{NaO}$ and achieved comparable results. They were also able to simultaneously trans-esterify glycerides while forming ammonium salts of the FFA(s), which upon injection in the GC inlet were esterified in a single preparation step. Again, this provided a significant advantage over existing procedures, which required FFA(s) and glycerides to be derivitized and analysed separately. Later, Metcalffe and Wang [32] also used TMAH in methanol in a single step process on different lipid mixtures in diethyl ether. This resulted in different phases where the transesterified methyl esters of the glycerides (FA) were contained in the organic phase, and the ammonium salts of the FFA(s) were in the aqueous phase. Conveniently, each phase was suitable for direct injection to a GC for FFA or glyceride (FA) characterization. This approach was later applied for the quantification of FFA in milk and cheese [33-36]. Martínez-Castro et al. [33] also investigated the effect of making the reaction mixture neutral, prior to the analysis which is usually recommended for GC analysis to protect the column and any PUFA(s) that may be present. They discovered that neutralizing the basic TMAH solution resulted in losses of SCFFA(s) and increased standard deviations in the analytical data. They attributed this to the dissociation of the ammonium salts at the selected $\mathrm{pH}$ (7.5-8.0). An advantage of TMAH is that when pyrolysed it degrades to TMA and methanol [31], which are highly volatile and thus suitable for GC analysis. However, TMA was also reported to interfere with peak determination [34]. This approach has obvious benefits in that both the FFA and the triglyceride components can be analysed from a sample in a single extraction; however, a limitation of the procedure using the TMAH reaction was highlighted by Martínez-Castro et al. [33]. These authors noted that some FA(s) from the organic glyceride layer were detected in the aqueous FFA layer, resulting in an overestimation of the FFA content in cheese. To overcome this, the authors separated each layer and washed with an appropriate solvent before analysis. Chavarri et al. [36] identified that if there is a large triglyceride to FFA ratio (which is the case in most dairy products), the issue with FFA dissociation is even more pronounced. The authors concluded that it was necessary to isolate FFA from the lipid mixture before employing the TMAH extraction/esterification method to alleviate this error.

\subsection{Direct on-column addition FFA}

The isolation of FFA(s) by aminopropyl solid phase extraction (SPE) columns followed by GC-FID analysis is widely used to quantify FFA [37-41], because the isolation process can be automated to a degree and is relatively simple to perform. Overall, it is a convenient alternative to derivatizition and as mentioned was developed by Kaluzny et al. [20] and subsequently improved $[13,37]$. The approach works without the need for derivatizition because FFA(s) are volatile and thus can be vapourised in a heated injection port. A cold on-column injection is employed followed by a programmed temperature ramp of the injector, as this allows for the increased separation of FFA(s) based on their volatility within the injector. There are also commercially available columns with specific free fatty acid phases (FFAP) that achieve complete separation of FFA(s) of chain lengths from $C_{2: 0}$ to $C_{22: 0 .}$. However, the acidic extracts reduce column performance and the high affinity of the FFA to the column phase can lead to irreversible adsorption, peak tailing, ghost peaks, or double peak formation [42, 43]. 


\subsection{Comparison of direct on-column addition FFA and TMAH FAME methods}

Chavarri et al. [36] compared the TMAH extraction esterification procedure to an on-column chromatographic procedure described by De Jong and Badings [13], where FFAs were isolated using aminopropyl columns before direct injection to GC-FID. Substantial discrepancies were evident between both methods in the analysis of FFA in Cheddar cheese. Levels of FFA were much higher using the on-column direct injection method (4007 ppm) than the TMAH method (1683 ppm). Typically, levels of FFA in Cheddar cheese are below 2000 ppm, thus a considerable error existed with the on-column direct injection method. The higher FFA levels were subsequently shown to be due to the dissociation of glycerides into the FFA layer. This can be rectified by isolating the FFA from the lipid mixture before employing the TMAH extraction/esterification. Mannion et al. [44] also compared and validated both methods. They employed an identical fat extraction and FFA isolation technique for both methods, using the diethyl ether/heptane procedure described by De Jong and Badings [13]. As well as, investigating analytical robustness accuracy, precision, limits of detection (LOD) and limits of quantification (LOQ) were also assessed. A wide range of dairy samples were analysed to assess method suitability to quantify FFAs in dairy products that have different sample matrices, lipid composition and FFA concentration. The products investigated ranged from cheeses (Cheddar, Brie and Blue Stilton), whole milk powder, infant formula, milk, yogurt, ice cream and enzyme-modified cheeses. Repeatability was expressed as percent (\%) relative standard deviation (RSD). Recoveries were assessed by spiking a known amount of FFA(s) into each sample with calculations based on recovery of the FFA between spiked and unspiked samples. The FFA(s) levels measured ranged from 173 ppm in infant formula to $126,615 \mathrm{ppm}$ in enzyme-modified cheese. Both methods provided similar results for each sample type, and repeatability was excellent (0.8-13.8\% RSD) except for milk, ice cream and yogurt (up to $46.2 \%$ RSD). Diethyl ether/heptane was used as the extracting solvent for both methods and is not suitable to reliably extract FFA(s) where the MFGM remains intact. This is easily remedied using an alternate ethanol-based extraction as described by De Jong and Badings [13] or De Jong et al. [37]. Analysis of FFA(s) directly as acids using the direct on-column addition method resulted in issues with column degradation due to the acidic nature of the FFA extract. This resulted in retention time peak shifts, peak broadening, and loss of resolution overtime. Additional steps were also required to prevent and monitor carryover due to the high affinity of FFA with the column phase. Analysis of the FFA with the TMAH FAME method was also not without issues. The most volatile SCFFA eluted with the solvent peak which impacted on sensitivity, and artefact formation periodically interfered with the quantification of other SCFFA. Overall, the direct on-column method proved to be the most sensitive, with an LOD of $0.7 \mathrm{ppm}$ and an LOQ of $3 \mathrm{ppm}$ and required less sample preparation as the FFAs were injected directly after isolation without the need for derivitization. Although the TMAH FAME method was the less sensitive of the two methods (LOD of $5 \mathrm{ppm}$; LOQ of $20 \mathrm{ppm}$ ), it proved to be a much more robust method where column integrity was not affected during analysis and retention times, and peak chromatography remained stable. In addition, the authors described an automated procedure for the dervitization which was significantly faster, used reduced solvent volumes than traditional procedures. 


\subsection{Future developments in GC-FID methods}

The use of higher molecular weight alcohols may be considered an alternative to overcome many issues experienced with methyl esters such as co-elution of solvent peaks with the most volatile FAME(s) and artefact formation. Studies by Parodi [45] demonstrated increased recoveries of SCFFA(s) when butyl esterification was carried out instead of methyl esterification. Parodi [45] evaluated several different methylation methods such as using $\mathrm{BF}_{3}$ in methanol [46], $\mathrm{CH}_{3} \mathrm{NaO}$ [47] and butylation methods using $\mathrm{H}_{2} \mathrm{SO}_{4}$ in butanol [48], di-n-butyl carbonate [49] and $\mathrm{BF}_{3}$ in butanol [45] to determine the fatty acid composition of butter fat. Parodi [45] expressed the data as ratio amounts of each fatty acid ester relative to the corresponding myristate ester and obtained better recovery when butyl esters were employed in comparison to $\mathrm{BF}_{3}$ in methanol or $\mathrm{CH}_{3} \mathrm{NaO}$ methylation methods. Parodi [50] utilised $\mathrm{KOH}$ in butanol to form butyl esters and was a modification of the methods described by Christopherson and Glass [25], and Kim Ha and Lindsay [51] where they utilised $\mathrm{BF}_{3}$ in butanol to quantify FFA in milk and cheese. Thus, employing butyl esters may be a more suitable alternative to methyl esters in the analysis of FFA in dairy products.

Most publications on the determination of FFA in dairy products reference long established extraction methods. Little or no development or validation seems to have taken place or has been published in recent years. Some efforts to improve and validate existing fat extraction methods have been undertaken. The widely used extraction method of Folch et al. [52] was modified by Firl et al. [53] to extract lipids from milk samples, which were subsequently converted to methyl esters using trimethylsulfonium hydroxide (TMSH) and analysed by GC-FID. They validated their approach by spiking milk samples with triglycerides and reported LODs, LOQs, accuracy and precision, which was particularly useful. However, quantification of FFA in dairy samples was not undertaken. Reis et al. [54] described a new method using thermal desorption to isolate FA in milk, using TMSH to convert the triglycerides into FAME(s). The reagent and milk samples were simply mixed into auto sampler vial and a heat-assisted reaction (a process they referred to as thermochemolysis) took place on initiation of the instrument sequence, with the FA quantified by GC mass spectrometry (MS). They compared this to the Rose-Gotlieb extraction method [55], where conventional transesterification using $\mathrm{KOH}$ in methanol had achieved comparable results in relation to recoveries and repeatability with the exception of $\mathrm{C}_{4: 0}$. These authors also reported limitations when dealing with raw nonhomogenised milk with poor repeatability between analyses. This was attributed to the volume of milk that was employed for the analysis not being representative of the entire milk sample. The evaluation of the THM technique was based on linearity, repeatability as a comparison against a conventional extraction method, FFA determination was not incorporated. Yurchenko et al. [56] performed a validated approach for the determination of FA(s) in bovine colostrum. They performed the extraction and preparation of FAME(s) as per the AOAC procedure [57], where methanolic $\mathrm{NaOH}$ followed by methanolic $\mathrm{BF}_{3}$ was added to the sample to form the FAME(s). These were subsequently extracted from the sample by phase separation using heptane and a saturated sodium chloride $(\mathrm{NaCl})$ solution. Linearity, accuracy, precision, LOD and LOQ were reported. There is scope for this method to be applied to milk and other dairy samples; however, individual determination of FFA(s) was not included, and only FA of carbon chain lengths $\mathrm{C}_{8: 0}-\mathrm{C}_{18: 0}$ were investigated. 
Amer et al. [58] described a new approach for the quantification of FFA(s) in milk by GC-MS. They used ethyl chloroformate to form ethyl esters insolution with pyridine added as a catalyst in chloroform. The recovery of deuterated FA internal standards was used to quantify each FFA. The authors also validated the method and reported repeatability, linearity, recoveries, LOD and LOQ. The method was applied to different bovine milk samples, raw, full fat $(3.55 \%)$, semi-skimmed $(1.5 \%)$ and skimmed milk $(0.1 \%)$. The stability of the method appears excellent with an RSD of $<4 \%$ for all FFA(s) and $>99 \%$ recoveries. Some of the issues experienced with volatility and water solubility of SCFFA(s) associated with methyl esters are overcome by the use of ethyl esters. In addition, the elimination of the requirement of prior extraction before the addition of the derivitizing agent is of some benefit. However, this method appears limited to aqueous samples only but appears a much more suitable alternative to many existing methods.

\section{Conclusion}

Despite the importance of FFA determination in dairy products for research, legislative, process development and quality control purposes, very little method development has been undertaken. In addition, little information on validation, analytical robustness, linearity, accuracy, LOD and LOQ was reported. Recent developments to improve extraction methods, where validation was carried out and reported, are a positive step forward [44, 52, 54, 58]. However, scope exists for further development to create methods that can rapidly, accurately and precisely quantify FFA(s) in a wide variety of dairy products in an efficient and robust manner.

\section{Author details}

Kieran N. Kilcawley* and David T. Mannion

*Address all correspondence to: kieran.kilcawley@teagasc.ie

Teagasc Food Research Centre, Co. Cork, Ireland

\section{References}

[1] Jensen RG, Ferris AM, Lammi-Keefe CJ. The composition of milk fat. Journal of Dairy Science. 1991;74(9):3228-3243

[2] Collins YF, McSweeney PLH, Wilkinson MG. Lipolysis and free fatty acid catabolism in cheese: A review of current knowledge. International Dairy Journal. 2003;13(11):841-866

[3] Jensen RG. The composition of bovine milk lipids: January 1995 to december 2000. Journal of Dairy Science. 2001;85(2):295-350 
[4] Taylor MW, MacGibbon AKH. Lipids I fatty acids a2 - Roginski, Hubert. Encyclopedia of Dairy Sciences. Oxford: Elsevier; 2002. pp. 1550-1554

[5] Christie WW. Composition and structure of milk lipids. In: Fox PF, editor. Advanced Dairy Chemistry Volume 2 Lipids. London, UK: Chapman and Hall; 1995. pp. 1-36

[6] Markiewicz-Keszycka M, Czyzak-Runowska G, Lipinska P, Wojtowski J. Fatty acid profile-a review. Bulletin of the Vetinary Institute in Pulawy. 2013;57:135-139

[7] Fox PF, Wallace JM. Formation of flavour compounds in cheese. Advances in Applied Microbiology. 1997;45:17-85

[8] Kamath S, Wulandewi A, Deeth H. Relationship between surface tension, free fatty acid concentration and foaming properties of milk. Food Research International. 2008;41(6): 623-629

[9] Kuzdzal-Savoie S. Determination of free fatty acids in milk and milk products. IDF Bulletin. 1980;118:53-66

[10] Gray IK. The quantitative analysis of free fatty acids in dairy products. New Zealand Journal of Dairy Science and Technology. 1975;10:158-162

[11] Salih AMA, Anderson M, Tuckley B. The determination of short- and long-chain free fatty acids in milk. Journal of Dairy Research. 1977;44(03):601-605

[12] Deeth HC, Fitzgerald CH, Snow AJ. A gas chromatographic method for the quantitative determination fo free fatty acids in milk and milk products. New Zealand Journal of Dairy Science and Technology. 1983;18:13-20

[13] De Jong C, Badings HT. Determination of free fatty acids in milk and cheese procedures for extraction, clean up, and capillary gas chromatographic analysis. Journal of High Resolution Chromatography. 1990;13(2):94-98

[14] IDF. Determination of indiviual free fatty acids - reference method. IDF Bulletin no 265. Brussels, Belgium: International Dairy Federation. 1991

[15] Harper WJ. Direct determination of acetic, propionic, and butyric acids in cheese Journal of Dairy Science. 1953;36(8):808-816

[16] Woo AH, Lindsay RC. Rapid method for quantitative analysis of individual free fatty acids in cheddar cheese. Journal of Dairy Science. 1982;65(7):1102-1109

[17] Needs EC, Ford GD, Owen AJ, Tuckley B, Anderson M. A method for the quantitative determination of individual free fatty acids in milk by ion exchange resin adsorption and gas-liquid chromatography. Journal of Dairy Research. 1983;50(03):321-329

[18] McNeill GP, O'Donoghue A, Connolly JF. Quantification and identification of flavour components leading to lipolytic rancidity in stored butter. Irish Journal of Food Science and Technology. 1986;10(1):1-10 
[19] McNeill GP, Connolly JF. A method for the quantification of individual free fatty acids in cheese: Application to ripening of cheddar-type cheeses. Irish Journal of Food Science and Technology. 1989;13(2):119-128

[20] Kaluzny MA, Duncan LA, Merritt MV, Epps DE. Rapid separation of lipid classes in high yield and purity using bonded phase columns. Journal of Lipid Research. 1985;26(1):135-140

[21] Woo AH, Lindsay RC. Development and characterization of an improved silicic acidkoh arrestant column for routine quantitative isolation of free fatty acids. Journal of the American Oil Chemists Society. 1980;57(12):414-416

[22] Stark W, Urbach G, Hamilton JS. Volatile compounds in butter oil: Iv. Quantitative estimation of free fatty acids and free $\delta$-lactones in butter oil by cold-finger molecular distillation. Journal of Dairy Research. 1976;43(03):469-477

[23] ISO 15884/IDF 182. Milk fat preparation of fatty acid methyl esters. Genova, Switzerland: International Standard Organisation and Brussels, Belgium: International Dairy Federation; 2002.

[24] Christie WW. Preparation of ester deriviataves of fatty acids for chromatographic analysis In: Christie WW, editor. Advances in lipid methodology 2. Dundee: Oily Press; 1993. pp. 69-111

[25] Christopherson SW, Glass RL. Preparation of milk fat methyl esters by alcoholysis in an essentially nonalcoholic solution. Journal of dairy science. 1969;52(8):1289-1290

[26] Luddy F, Barford R, Herb S, Magidman P. A rapid and quantitative procedure for the preparation of methyl esters of butteroil and other fats. Journal of the American Oil Chemists Society. 1968;45(8):549-552

[27] Prelog V, Plantanida M. Eine methode zur darstellung von methylestern schwer veresterbarer säuren. Hoppe-Seyler' s Zeitschrift für physiologische Chemie. 1936;244(1-2):56-58

[28] Robb EW, Westbrook JJ. Preparation of methyl esters for gas liquid chromatography of acids by pyrolysis of tetramethylammonium salts. Analytical Chemistry. 1963;35(11):1644-1647

[29] Downing DT. Analysis of aqueous solutions of organic acids by pyrolysis of their tetramethylammonium salts in the gas chromatograph. Analytical Chemistry. 1967;39(2): 218-221

[30] Downing DT, Greene RS. Methylation of fatty acids by pyrolysis of their tetramethylammonium salts in the gas chromatograph. Analytical Chemistry. 1968;40(4):827-828

[31] McCreary DK, Kossa WC, Ramachandran S, Kurtz RR. A novel and rapid method for the preparation of methyl esters for gas chromatography: Application to the determination of the fatty acids of edible fats and oils. Journal of Chromatographic Science. 1978;16(8):329-331 
[32] Metcalffe L, Wang C. Rapid preparation of fatty acid methyl esters using organic basecatalyzed transesterification. Journal of Chromatographic Science. 1981;19(10):530-535

[33] Martínez-Castro I, Alonso L, Juárez M. Gas chromatographic analysis of free fatty acids and glycerides of milk fat using tetramethylammonium hydroxide as catalyste. Chromatographia. 1986;21(1):37-40

[34] Martin-Hernández MC, Alonso L, Juárez M, Fontecha J. Gas chromatographic method for determining free fatty acids in cheese. Chromatographia. 1988;25(2):87-90

[35] Juárez M, de la Fuente MA, Fontecha J. Improved gas chromatographic method for the determination of the individual free fatty acids in cheese using a capillary column and a ptv injector. Chromatographia. 1992;33(7-8):351-355

[36] Chavarri F, Virto M, Martin C, Najera AI, Santisteban A, Barron LJR, et al. Determination of free fatty acids in cheese: Comparison of two analytical methods. Journal of Dairy Research. 1997;64(03):445-452

[37] De Jong C, Palma K, Neeter R. Sample preparation before capillary gas-chromatographic estimation of free fatty acids in fermented dairy products. Netherlands Milk and \& Dairy Journal 1994;48:151-156

[38] Kilcawley KN, Nongonierma AB, Hannon JA, Doolan IA, Wilkinson MG. Evaluation of commercial enzyme systems to accelerate cheddar cheese ripening. International Dairy Journal. 2012;26(1):50-57

[39] Hickey DK, Kilcawley KN, Beresford TP, Sheehan EM, Wilkinson MG. Starter strain related effects on the biochemical and sensory properties of cheddar cheese. Journal of Dairy Research. 2007;74(1):9-17

[40] Hickey DK, Kilcawley KN, Beresford TP, Sheehan EM, Wilkinson MG. The influence of a seasonal milk supply on the biochemical and sensory properties of cheddar cheese. International Dairy Journal. 2006;16(6):679-690

[41] Calzada J, del Olmo A, Picon A, Nuñez M. Effect of high-pressure-processing on lipolysis and volatile compounds of brie cheese during ripening and refrigerated storage. International Dairy Journal. 2014;39(2):232-239

[42] Emery EM, Koerner WE. Double-column programmed temperature gas chromatography. Volatile polar column packings and quantitative aspects. Analytical Chemistry. 1962;34(10):1196-1198

[43] Ackman RG, Burgher RD. Quantitative gas liquid chromatographic estimation of volatile fatty acids in aqueous media. Analytical Chemistry. 1963;35(6):647-652

[44] Mannion DT, Furey A, Kilcawley KN. Comparison and validation of 2 analytical methods for the determination of free fatty acids in dairy products by gas chromatography with flame ionization detection. Journal of Dairy Science. 2016;69(7):5047-5063 
[45] Parodi PW. A study of ester preparation for the gas chromatographic analysis of the fatty acids composition of butterfat. Australian Journal of Dairy Technology. 1967;22:144-145

[46] Metcalfe LD, Schmitz AA, Pelka JR. Rapid preparation of fatty acid esters from lipids for gas chromatographic analysis. Analytical Chemistry. 1966;38(3):514-515

[47] DeMan JM. Determination of the fatty acid composition of milk fat by dual column temperature programmed gas-liquid chromatography. Journal of Dairy Science. 1964;47(5):546-547

[48] Gander GW, Jensen RG, Sampugna J. Analysis of milk fatty acids by gas-liquid chromatography. Journal of Dairy Science. 1962;45(3):323-328

[49] Sampugna J, Pitas RE, Jensen RG. Gas-liquid chromatographic analysis of milk fat: Rapid preparation of butyl esters. Journal of Dairy Science. 1966;49(11):1462-1463

[50] Parodi P. Fatty acid composition of australian butter and milk fats. Australian Journal of Dairy Technology. 1970;25:200-205

[51] Kim Ha J, Lindsay RC. Method for the quantitative analysis of volatile free and total branched-chain fatty acids in cheese and milk fat. Journal of Dairy Science. 1990;73(8): 1988-1999

[52] Folch J, Lees M, Stanley GHS. A simple method for the isolation and purification of total lipides from animal tissues. Journal of Biological Chemistry. 1957;226(1):497-509.

[53] Firl N, Kienberger H, Rychlik M. Validation of the sensitive and accurate quantitation of the fatty acid distribution in bovine milk. International Dairy Journal. 2014;35(2):139-144

[54] Reis MG, dos Reis MM, Leath S, Stelwagen K. Direct analysis of fatty acid profile from milk by thermochemolysis-gas chromatography-mass spectrometry. Journal of Chromatography A. 2011;1218(2):316-323

[55] Walstra P, Mulder H. Gravimetric methods for the determination of fat content of milk and milk products Netherlands Milk and \& Dairy Journal. 1962;16:172-184

[56] Yurchenko S, Sats A, Poikalainen V, Karus A. Method for determination of fatty acids in bovine colostrum using GC-FID. Food Chemistry. 2016;212:117-122

[57] AOAC. Methods of analysis. 96933 Fatty acids in oils and fats (17th edition). Virginia, USA. 2000

[58] Amer B, Nebel C, Bertram HC, Mortensen G, Hermansen K, Dalsgaard TK. Novel method for quantification of individual free fatty acids in milk using an in-solution derivatisation approach and gas chromatography-mass spectrometry. International Dairy Journal. 2013;32(2):199-203 